\title{
Impact of percutaneous patent foramen ovale closure on migraine headaches in patients with history of ischemic neurological events
}

\author{
Przemysław Węglarz ${ }^{1}$, Katarzyna Spisak-Borowska², Tomasz Bochenek ${ }^{3}$, Ewa Konarska-Kuszewska³, \\ Jerzy Machowski ${ }^{2}$, Maria Trusz-Gluza ${ }^{3}$, Grzegorz Bajor ${ }^{1}$, Katarzyna Mizia-Stec ${ }^{3}$, Joel P. Giblett ${ }^{4}$, \\ Patrick A. Calvert ${ }^{4}$
}

${ }^{1}$ Department of Human Anatomy, Medical University of Silesia, Katowice, Poland

${ }^{2}$ Neurology Department, Oswiecim Hospital, Oswiecim, Poland

${ }^{3}$ Department of Cardiology, Medical University of Silesia, Katowice, Poland

${ }^{4}$ Department of Cardiology, Royal Papworth Hospital, Cambridge, United Kingdom

Adv Interv Cardiol 2020; 16, 3 (61): 315-320

DOI: https://doi.org/10.5114/aic.2020.99267

\section{A bstract}

Introduction: Observational studies have shown that migraine has been associated with patent foramen ovale (PFO). Whilst studies investigating PFO closure for the treatment of migraine have been neutral, there is some evidence that symptoms of migraine may improve if the PFO was closed after ischemic stroke.

Aim: To establish whether closure of PFO in patients with stroke or transient ischemic attack (TIA) is associated with reduction in the severity of co-existent migraine headaches.

Material and methods: Patients with ischemic stroke or TIA, PFO suitable for percutaneous closure and migraine, were given migraine severity questionnaires prior to PFO closure. These were followed up at 6 and 12 months after closure with the same questionnaire. The primary endpoint was change in migraine severity using the Migraine Severity Scale (MIGSEV). Migraine episode frequency, disability (using the MIDAS scale), and pain intensity were also assessed.

Results: Sixty-two patients were included in the analysis. MIGSEV scores reduced from 7 (7-8) at baseline to 4 (3.25-6) at 6-month follow-up, and $3(0-4)$ at 12 -month follow-up $(p<0.001)$. Other measures of migraine headache were also improved at both 6-and 12-month follow-up. Twenty-four (38\%) patients were rendered migraine free at 12 months.

Conclusions: PFO closure for stroke or TIA prevention in patients with migraine was associated with a reduction in markers of migraine headache severity.

Key words: migraine, headache, patent foramen ovale, stroke, patent foramen ovale closure.

\section{S u m m a ry}

Patent foramen ovale is frequently associated with migraine but it is uncertain whether closure improves symptoms. This observational study evaluated patients with migraine and closure for cryptogenic stroke. It showed marked and sustained improvement in migraine symptoms.

\section{Introduction}

Migraine is a common condition associated with substantial morbidity and a significant economic impact. Migraines affect $13 \%$ of the population aged 20-64 years with an increased prevalence amongst young adults ( $90 \%$ of patients experiencing their first migraine before the age of 35) and higher prevalence in females [1, 2]. Large numbers of scientific reports refer to the coexistence of patent foramen ovale (PFO), stroke and migraine [3]. One study demonstrated that $50 \%$ of patients with cryptogenic stroke had migraine symptoms, and of these $79 \%$ had a PFO with right to left shunt [4]. PFO closure

Corresponding author:

Dr Joel Peter Giblett, Department of Cardiology, Royal Papworth Hospital, Cambridge, United Kingdom, e-mail: joel.giblett@doctors.org.uk Received: 14.02.2020, accepted: 24.06.2020. 
is recommended for the prevention of ischaemic stroke in patients with PFO and a history of cryptogenic stroke $[5,6]$. Previous studies showed that PFO closure might be beneficial in migraine patients by reducing migraine attacks and migraine days, especially in patients whose majority of migraine attacks are with aura [7, 8].

PFO is found in about $40-60 \%$ of people who have migraine with aura compared with $20-30 \%$ of people with migraine without aura and the general population [9, 10]. Results of the randomized clinical trials PRIMA and PREMIUM, in which patients with PFO and migraine underwent percutaneous PFO closure, were neutral for their primary endpoints [11, 12]. However, they demonstrated a significantly higher number of patients free from migraine with aura in the PFO closure groups.

\section{Aim}

This prospective observational study aimed to assess the effectiveness of PFO closure on migraine symptoms, in patients undergoing closure for stroke or transient ischemic attack.

\section{Material and methods}

\section{Study population}

All patients presenting with transient ischemic attack (TIA) or stroke at a single institution were screened for PFO. Those patients with a confirmed PFO were screened for enrolment in the study. Patients with a confirmed diagnosis of migraine according to the diagnostic criteria of the International Classification of Headache Diseases (ICHD2), evidence of cerebral infarction or at least two TIAs, presence of a PFO on transesophageal echocardiography (TEE), and capacity to consent to the study were included. Exclusion criteria included patients with a poorly documented or short history ( $<12$ months) of migraine, a clear cause of cerebral infarction other than the PFO, contraindications to PFO closure, or a life expectancy of less than 2 years. Evidence of stroke or TIA was adjudicated by a neurologist based on all available clinical data, including history, physical examination, computed tomography (CT) and magnetic resonance imaging (MRI) where available.

\section{Evaluation of patent foramen ovale}

Patent foramen ovale was diagnosed using contrast TEE, which was part of the screening for enrolment in the study, and usual medical care in the institution for patients with cryptogenic stroke. All TEE were performed and analyzed by the same echocardiographer. The intra-atrial septum was assessed in mid-esophageal four chamber, mid-esophageal short axis, and mid-esophageal bicaval views. Intravenous contrast $(9 \mathrm{ml}$ of $0.9 \%$ saline solution with $1 \mathrm{ml}$ of air, agitated between two connected $10 \mathrm{ml}$ syringes) was administered and PFO was assessed using Valsalva maneuvers. The examinations were evaluated on a scale between 0 and 3 , with 0 meaning no bubbles of contrast crossing into the left atrium and 3 meaning a large cloud of bubbles [13]. Atrial septum aneurysm (ASA) was diagnosed when deviation of septum of at least $10 \mathrm{~mm}$ to the right or left atrium was observed. Amplitude of deviation was characterized as less than $10 \mathrm{~mm}, 10-15 \mathrm{~mm}$, or more than $15 \mathrm{~mm}$.

\section{Evaluation of migraine symptoms}

Migraine was diagnosed by a neurologist prior to inclusion in the study by means of the ICHD2 criteria [14]. Patients included in the study undertook a migraine questionnaire prior to PFO closure, and at 6 and 12 months after the procedure. This covered frequency, duration, intensity and location of pain, associated symptoms, triggers, nature, aura if present, and analgesia use. Migraine Severity Scale (MIGSEV) and Migraine Disability Assessment Test (MIDAS) scores were recorded $[15,16]$. A MIGSEV and MIDAS score of 0 at follow-up was defined as complete resolution of migraine. A reduction in either score at follow-up of $\geq 1$ point in the MIGSEV scale, and $\geq 2$ points in the MIDAS questionnaire was considered an improvement. All patients were allowed to use their previously used analgesics.

\section{Patent foramen ovale closure}

All the PFO closure procedures were performed by a single operator using local anesthesia and conscious sedation as required. Procedures were performed from the femoral vein, puncture with a short $6 \mathrm{~F}$ sheath. The PFO channel was cannulated with a multipurpose diagnostic catheter with fluoroscopic and TEE guidance. At least 5000 IU of heparin was administered via the sheath. The occluder was advanced in the transseptal sheath and implanted on the septum, guided with fluoroscopy and TEE. Cardia Intrasept (Cardia, MN, USA) and Occlutech Figula (Occlutech, Germany) devices were implanted. Patients were routinely admitted for 3-4 days. In follow-up patients received dual antiplatelet therapy with aspirin and clopidogrel for 6 months, followed by aspirin monotherapy for the remainder of the study. Patients were not anticoagulated during the study. Patients were followed up with transthoracic echocardiography, including intravenous contrast administration and a Valsalva maneuver, at 6 and 12 months.

\section{Statistical analysis}

Data were gathered prospectively but the statistical analysis plan was written after data collection. The primary outcome measure in the study was change in migraine severity assessed on the MIGSEV scale. Secondary endpoints included change in MIDAS score, duration of pain, pain intensity, and number of migraine attacks per month. A sample size calculation was not performed for 


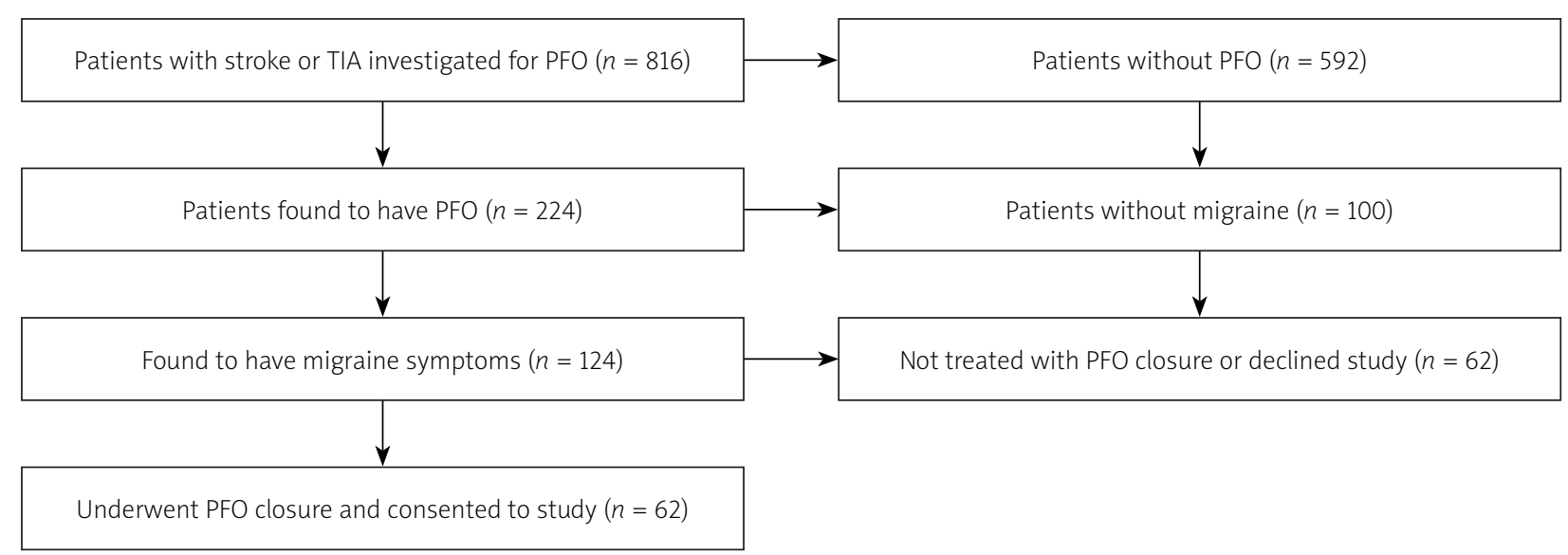

Figure 1. Study flowchart

this observational study. Since endpoints were ordinal they were analyzed with the Friedman test, a non-parametric test which allows comparison across multiple timepoints. A further post hoc analysis comparing changes between individual timepoints was also undertaken. Comparisons between those patients with and without aura were performed using the Mann-Whitney $U$ test for continuous data and the $\chi^{2}$ test for categorical data. In this study a $p$-value of $<0.05$ was considered to be statistically significant. Data are presented as median (interquartile range) or $n$ (\%).

The study was considered by the Bioethics Committee of the Silesian University of Medicine (No. KNW/0022/ $\mathrm{KB} / 146 / 16)$. The British authors of this manuscript assisted with analysis of the data, drafting of the manuscript and critical review of its content.

\section{Results}

\section{Study population and procedure}

The study population is highlighted in the study flow chart (Figure 1). Between 2003 and 2014, 816 patients presenting with a TIA or stroke were investigated for the presence of a PFO. A PFO was confirmed in 224 patients, of whom 124 were found to have co-existing migraine symptoms, and 149 patients went on to have a PFO closure procedure. Of these patients 62 with both migraine and PFO closure consented to be included in the study.

Patient characteristics are included in Table I. Overall, most patients were female, with low frequency of conventional risk factors for ischemic stroke. Most patients had a PFO with a large degree of leak, with an atrial septal aneurysm. All patients had migraine symptoms after the stroke and prior to PFO closure. The majority of patients in the study had migraine that was associated with an aura (74\%). Medications used by patients on an ad hoc basis included paracetamol (38\%), triptans (27\%), aspirin (19\%), ibuprofen (11\%) and ergotamine (11\%). Prophylactic treatments were dominated by topiramate (4.8\%), sodium valproate (1.6\%) and amitriptyline (1.6\%).
PFO closure was performed in all patients included in the study. Median time from stroke to PFO closure was 147 days (91-193). The most common device was the Cardia Intrasept, deployed in 47 (76\%) patients. No major periprocedural complications were reported. A single patient suffered a hematoma, treated with manual

Table I. Clinical and anatomical characteristics of patients included in the study $(n=62)$

\begin{tabular}{|c|c|}
\hline Parameter & Results \\
\hline \multicolumn{2}{|l|}{ Clinical characteristics: } \\
\hline Age [years] & $34(26-46)$ \\
\hline Male sex & $11(17.7)$ \\
\hline Hypertension & $10(16.1)$ \\
\hline Diabetes & $0(0)$ \\
\hline Smoking & $13(20.9)$ \\
\hline Hypercholesterolemia & $14(22.6)$ \\
\hline Atrial fibrillation & $0(0)$ \\
\hline Varicose veins of the lower extremities & $8(12.9)$ \\
\hline Oral contraception & $\begin{array}{c}10(19.6, \\
\% \text { of female sex })\end{array}$ \\
\hline Aspirin use & $12(19)$ \\
\hline Migraine with aura, MA(+) & $46(74.2)$ \\
\hline \multicolumn{2}{|l|}{ Anatomical characteristics: } \\
\hline Presence of atrial septal aneurysm & $44(71.0)$ \\
\hline Presence of Chiari network & $14(22.6)$ \\
\hline \multicolumn{2}{|c|}{ Degree of leak (transesophageal echocardiography): } \\
\hline Small & $5(8.0)$ \\
\hline Medium & $22(35.5)$ \\
\hline Large & $35(56.5)$ \\
\hline
\end{tabular}

All values are median (IQR), or $n(\%)$. 
Table II. Change in endpoints at follow-up

\begin{tabular}{lcccc} 
Endpoint & Baseline & 6-month follow-up & 12-month follow-up & $P$-value \\
\hline MIGSEV Scale & $7(7-8)$ & $4(3.25-6)$ & $3(0-4)$ & $<0.001$ \\
\hline MIDAS Scale & $24(20-26)$ & $11(5.25-15)$ & $8(0-10)$ & $<0.001$ \\
\hline Migraine [episodes/month] & $6(5-7)$ & $2(1.25-5)$ & $2(0-2)$ & $<0.001$ \\
\hline Pain intensity (0-10) & $7(6-7)$ & $3(2-4)$ & $2(0-2)$ & $<0.01$ \\
\hline Pain duration [h] & $12(8.5-24)$ & $4(0-4)$ & $3(0-4)$ & $<0.001$
\end{tabular}

P-value is Friedman test for change across the three timepoints.

pressure, that precipitated a prolonged hospital stay, but there were no long-term sequelae. At 6-month follow-up $5(8.1 \%)$ patients had a significant residual shunt. This was reduced to $1(1.6 \%)$ patient at 12 months.

\section{Migraine relief}

Reductions in measures of migraine were recorded in the study. These are detailed in Table II. The primary endpoint of the study was reduction in the MIGSEV scale evaluated with a Friedman test across multiple endpoints. The score was reduced from 7 at baseline to 4 and 3, at 6-month and 12-month follow-up respectively. Other endpoints were similarly reduced. Improvements in endpoints are presented visually in Figure 2. Post hoc analysis showed that all endpoints improved between 0 and 6 months $(p<0.001)$, whilst all except duration of pain significantly improved between 6 and 12 months $(p<0.01)$. Complete resolution of migraine (based on MIDAS and MIGSEV scores) was recorded in 14 (22.5\%) patients at 6-month follow-up and 24 (38.7\%) after 12 months, with $89 \%$ of patients reporting either resolution or significant improvement. The median duration of pain decreased from $12(8.5-24) \mathrm{h}$ before the procedure to $3(2-4)$ h at 6 months and $2(0-3)$ h at 12 months after

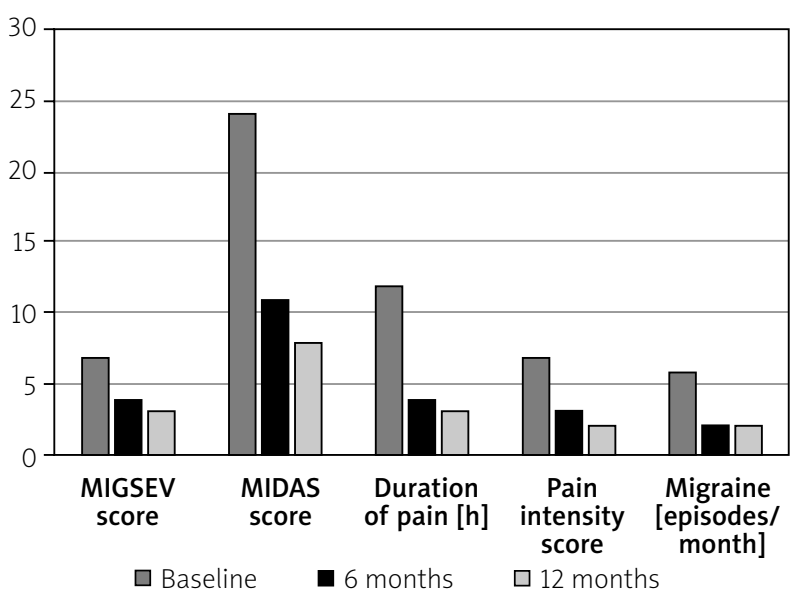

Figure 2. Primary and secondary endpoints at baseline, 6- and 12-month follow-up. All changes are significant $(p<0.01)$ on Friedman test for change over multiple timepoints
PFO closure. There were also reductions in pain intensity and number of attacks per month. Prior to the procedure all patients in the study needed to use as required analgesia for headache symptoms. This was reduced to $58 \%$ at 12-month follow-up $(p<0.001)$. Twenty-three patients reporting complete resolution at 12 months had migraine with aura whilst only a single patient without aura reported complete resolution. Since this was not a prespecified analysis it should be considered a hypothesis-generating finding. No patients without aura reported new symptoms after closure of the PFO.

\section{Discussion}

This study has demonstrated that, in a cohort of patients suffering migraine undergoing PFO closure for secondary prevention after cryptogenic stroke, closure was associated with a statistically significant reduction in the frequency and severity of migraine symptoms.

The reason for the co-occurrence of migraine and PFO remains unclear. Shunting of blood from the venous to arterial circulation allows various factors (vasoactive substances, platelet activating factors or amines) to bypass the natural filter of the pulmonary circulation. This allows them to achieve higher concentrations in the brain, potentially triggering migraine. Furthermore, microemboli, migrating through the PFO tunnel into the distal branches of cerebral arteries, may cause direct cerebral ischemia, or cortical spreading depression (believed to be the electrophysiological basis of aura), a factor in the pathogenesis of migraine headaches [17-19]. Observational studies have shown increased incidence of migraine in patients with PFO [20]. A meta-analysis showed that PFO is associated with a 3.4-fold increase in migraine-with-aura compared to those without a PFO [21]. Incidence of migraine appears to be influenced by the size of the leak through the PFO tunnel with case-control studies describing high incidence of large shunts in the migraine sufferers compared to the control subjects [20]. In this study, most patients had at least a medium shunt through the PFO tunnel (92\%). In patients with a combination of cryptogenic stroke and migraine, there is a high prevalence $(79 \%)$ of PFO with right-to-left shunt, and greater still when migraine with aura is considered (93\%) 
[22]. A study of divers with decompression sickness was the first demonstration that PFO closure may lead to improvement in migraine [23].

The first randomized trial dedicated to the analysis of PFO closure and its effect on migraine was the MIST study. This study showed no significant improvement in migraine symptoms for patients undergoing PFO closure compared to the control group [24]. The trial assessed patients with a low frequency of migraine. There were a large number of residual shunts, and the follow-up period was short. The MIST study did not include patients with a history of ischemic stroke or TIA as in this study. Other studies such as PREMIUM and PRIMA also failed to meet their primary endpoints, although there was a small reduction in headache $[11,12]$. It is worth noting that swapping the primary endpoints of these trials rendered both positive [25]. Two further randomized trials (MIST II and ESCAPE) designed to assess the effect of PFO closure on migraine were discontinued.

In this study, resolution of migraine after PFO closure was observed in $22.5 \%$ of patients at 6 months of follow-up, and $38.7 \%$ at 12 months. Resolution of migraine or improvement was reported by $88.7 \%$ of patients after 12 months of observation. These results indicate that in a selected population, percutaneous PFO closure is very effective in reducing the symptoms of migraine. The findings are consistent with previous observational studies [26-28]. The reasons for the additional late reduction in symptoms are not clear, but the authors speculate that some of the effect may be due to the additional closure of PFO channels, with reduced shunt. In another recently published observational study, residual shunt was associated with continuing symptoms [29]. Our finding that those with aura were more likely to have abolition of symptoms was also found in that retrospective study. Use of analgesia was significantly reduced in the study, which is also consistent with other studies. Another factor that may account for symptom improvement is the use of dual antiplatelet therapy after the procedure. Both aspirin and clopidogrel have been reported to improve migraine symptoms [30, 31]. According to study recommendations, both antiplatelet drugs were used for a period of 6 months, but 12-month follow-up reduced the likelihood that these drugs affected the results.

All the patients in this study would reasonably qualify for PFO closure on the basis of neurological symptoms related to cerebral ischemia. There is a need for further investigation of whether patients without stroke would benefit from PFO closure. In patients without stroke the benefit is less clear, and further randomized trials in highly symptomatic patients are needed.

This study was an observational study without a control group to compare the results to. There is a high risk of a placebo effect, from having a procedure performed, influencing the results of the study. The long follow-up period of the study was designed to limit a short-term placebo effect from the procedure. Headache symptoms are particularly vulnerable to the placebo effect, with up to $50 \%$ of treatment effects due to placebo in migraine studies [32]. In this study however, $88.7 \%$ of patients saw an improvement in their symptoms, suggesting that the benefit may exceed the placebo effect. It is worth considering that sham surgical procedures may have an increased placebo effect compared to pharmaceutical interventions [33]. Detailed information regarding medication use was not collected during the follow-up. Although there was a reduction in use of analgesia at 12 months, lack of granularity may also mask other changes in treatment, affecting the results. The authors believe this effect is likely to be small. The authors believe that the reductions seen in this study are clinically significant but acknowledge that the cut-offs included in the trial design were not formally validated prior to the study. Nonetheless, the absence of any migraine in such a high proportion of the population and the improvement in other endpoints of migraine severity suggest that these cut-offs are appropriate. No sample size calculation was undertaken before data collection as the data analysis was undertaken retrospectively, and the patients were recruited relatively slowly to this study. In addition, of patients apparently eligible, a high number of them were not recruited and there are no data to explain why they were not recruited. Many patients were recruited before the publication of randomized control trials confirming a benefit of PFO closure, meaning that many may have opted for anticoagulation alone. Despite these recruitment challenges, the results of the study should be taken together with other observational studies in this area, and these should serve as evidence supporting the need for a randomized clinical trial addressing this group.

\section{Conclusions}

Percutaneous PFO closure appears effective in reducing the severity of migraine in a selected population of patients who have a history of ischemic stroke. Further research directed at other groups who may benefit from PFO closure is needed.

\section{Conflict of interest}

Dr Calvert undertakes proctoring for Abbott Vascular and Occlutech. Other authors declare no conflict of interest.

\section{References}

1. Stewart WF, Simon D, Shechter A, Lipton RB. Population variation in migraine prevalence: a meta-analysis. J Clin Epidemiol 1995; 48: 269-80.

2. Burch RC, Loder S, Loder E, Smitherman TA. The prevalence and burden of migraine and severe headache in the United States: updated statistics from government health surveillance studies. Headache 2015; 55: 21-34. 
3. Ferrarini G, Malferrari G, Zucco R, et al. High prevalence of patent foramen ovale in migraine with aura. J Headache Pain 2005; 6: 71-6.

4. West BH, Noureddin N, Mamzhi Y, et al. Frequency of patent foramen ovale and migraine in patients with cryptogenic stroke. Stroke 2018; 49: 1123-8.

5. Araszkiewicz A, Bartuś S, Demkow M, et al. Interventional closure of patent foramen ovale in prevention of thromboembolic events. Consensus document of the Association of Cardiovascular Interventions and the Section of Grown-up Congenital Heart Disease of the Polish Cardiac Society. Kardiol Pol 2019; 77: 1094-105.

6. Giblett JP, Abdul-Samad O, Shapiro LM, et al. Patent foramen ovale closure in 2019. Interv Cardiol Rev 2019; 14: 34.

7. Hildick-Smith D, Williams TM. Patent foramen ovale and migraine headache. Interv Cardiol Clin 2017; 6: 539-45.

8. Elbadawi A, Barssoum K, Abuzaid AS, et al. Meta-analysis of randomized trials on percutaneous patent foramen ovale closure for prevention of migraine. Acta Cardiol 2019; 74: 124-9.

9. Tatlidede AD, Oflazoğlu B, Celik SE, et al. Prevalence of patent foramen ovale in patients with migraine. Agri 2007; 19: 39-42.

10. Carod-Artal F, Ribeiro L da S, Braga H, et al. Prevalence of patent foramen ovale in migraine patients with and without aura compared with stroke patients. A Transcranial Doppler Study. Cephalalgia 2006; 26: 934-9.

11. Mattle HP, Evers S, Hildick-Smith D, et al. Percutaneous closure of patent foramen ovale in migraine with aura, a randomized controlled trial. Eur Hear J 2016; 37: 2029-36.

12. Tobis JM, Charles A, Silberstein SD, et al. Percutaneous closure of patent foramen ovale in patients with migraine: the PREMIUM trial. J Am Coll Cardiol 2017; 70: 2766-74.

13. Mahmoud AN, Elgendy IY, Agarwal N, et al. Identification and quantification of patent foramen ovale-mediated shunts. Interv Cardiol Clin 2017; 6: 495-504.

14. Lipton RB, Bigal ME, Steiner TJ, et al. Classification of primary headaches. Neurology 2004; 63: 427-35.

15. El Hasnaoui A, Vray M, Blin P, et al.; HEMISHERE Study Group. Assessment of migraine severity using the MIGSEV scale: relationship to migraine features and quality of life. Cephalalgia 2004; 24: 262-70.

16. Stewart WF, Lipton RB, Dowson AJ, Sawyer J. Development and testing of the Migraine Disability Assessment (MIDAS) Questionnaire to assess headache-related disability. Neurology 2001; 56 (6 Suppl 1): S20-8.

17. Nozari A, Dilekoz E, Sukhotinsky I, et al. Microemboli may link spreading depression, migraine aura, and patent foramen ovale. Ann Neurol 2010; 67: 221-9.

18. Wammes-van der Heijden E, Tijssen C, Egberts A. Right-to-left shunt and migraine: the strength of the relationship. Cephalalgia 2006; 26: 208-13.

19. Finocchi C, Del Sette M. Migraine with aura and patent foramen ovale: myth or reality? Neurol Sci 2015; 36 Suppl 1: 61-6.

20. Schwerzmann M, Nedeltchev K, Lagger F, et al. Prevalence and size of directly detected patent foramen ovale in migraine with aura. Neurology 2005; 65: 1415-8.

21. Takagi H, Umemoto T; ALICE (All-Literature Investigation of Cardiovascular Evidence) Group. A meta-analysis of case-control studies of the association of migraine and patent foramen ovale. J Cardiol 2016; 67: 493-503.
22. West BH, Noureddin N, Mamzhi Y, et al. Frequency of patent foramen ovale and migraine in patients with cryptogenic stroke. Stroke 2018; 49: 1123-8.

23. Wilmshurst PT, Nightingale S, Walsh KP, Morrison WL. Effect on migraine of closure of cardiac right-to-left shunts to prevent recurrence of decompression illness or stroke or for haemodynamic reasons. Lancet 2000; 356: 1648-51.

24. Dowson A, Mullen MJ, Peatfield R, et al. Migraine Intervention With STARFlex Technology (MIST) trial: a prospective, multicenter, double-blind, sham-controlled trial to evaluate the effectiveness of patent foramen ovale closure with STARFlex septal repair implant to resolve refractory migraine. Circulation 2008; 117: 1397-404.

25. Meier B. Patent foramen ovele, good reasons to close it. Dtsch Med Wochenschr 2018; 143: 354-6.

26. Wahl A, Praz F, Tai T, et al. Improvement of migraine headaches after percutaneous closure of patent foramen ovale for secondary prevention of paradoxical embolism. Heart 2010; 96: 96773.

27. Dubiel M, Bruch L, Schmehl I, et al. Migraine headache relief after percutaneous transcatheter closure of interatrial communications. J Interv Cardiol 2008; 21: 32-7.

28. Araszkiewicz A, Grygier M, Iwańczyk S, et al. Long-term follow-up after percutaneous closure of patent foramen ovale with Amplatzer PFO occluder: a single center experience. Adv Interv Cardiol 2016; 12: 49-54.

29. Ben-Assa E, Rengifo-Moreno P, Al-Bawardy R, et al. Effect of residual interatrial shunt on migraine burden after transcatheter closure of patent foramen ovale. JACC Cardiovasc Interv 2020; 13: 293-302.

30. Wilmshurst PT, Nightingale S, Walsh KP, Morrison WL. Clopidogrel reduces migraine with aura after transcatheter closure of persistent foramen ovale and atrial septal defects. Heart 2005; 91: 1173-5.

31. Diener H, Hartung E, Chrubasik J, et al. A comparative study of oral acetylsalicyclic acid and metoprolol for the prophylactic treatment of migraine. a randomized, controlled, double-blind, parallel group phase III study. Cephalalgia 2001; 21: 120-8.

32. van der Kuy PHM, Lohman JJHM. A quantification of the placebo response in migraine prophylaxis. Cephalalgia 2002; 22: 265-70.

33. Holtedahl R, Brox JI, Tjomsland O. Placebo effects in trials evaluating 12 selected minimally invasive interventions: a systematic review and meta-analysis. BMJ Open 2015; 5: e007331. 